\title{
The lysozyme gene in fish
}

TAKASHI AOKI,* JUN-ICHI HIKIMA, SONOMI MINAGAWA, AND IKUO HIRONO

Laboratory of Genetics and Biochemistry, Department of Aquatic Biosciences, Tokyo University of Fisheries, 4-57 Konan, Minato-ku, Tokyo 108-8477, Japan (aoki@tokyo-u-fish.ac.jp).

\section{KEY WORDS: Lysozyme, Innate immunity, Gene structure, Molecular evolution, Lytic activity}

\section{INTRODUCTION}

Lysozyme (muramidase, EC.3.2.1.17) is a widely distributed enzyme, which are located in the serum, mucus and many other tissues of fish and higher vertebrates. It catalyzes the hydrolysis of bacterial cell walls, and acts as a non-specific innate immunity molecule against the invasion of bacterial pathogens. ${ }^{1,2)}$ According to their amino acid sequences, lysozyme are classified into 5 types: chicken-type lysozyme (c-type), goose-type lysozyme (g-type), plant lysozyme, bacterial lysozyme and T4 phage lysozyme (phage-type). ${ }^{2,45}$ In fish, only cDNA sequences of two c-type lysozymes from rainbow trout have previously been reported. ${ }^{6)}$ Recently, we have cloned and characterized the c-type and g-type lysozyme genes from Japanese flounder, Paralichthys olivaceus. ${ }^{3,7,8)}$ This paper summarizes their gene structure, gene evolution, and gene expression and activity.

\section{FLOUNDER C-TYPE LYSOZYME GENE}

The fish c-type lysozyme gene sequence from Japanese flounder, ${ }^{3)}$ consists of $3,617 \mathrm{bp}$, including 4 exons and 3 introns (Fig. 1). Structural comparison of the c-type lysozyme genes from other vertebrates and Japanese flounder indicate a correspondence in the positions of the exons and catalytic residues (Glu 35 and Asp 52). Hikima et al. ${ }^{3)}$ have found 45 novel repeated sequences (approx. $1.4 \mathrm{~kb}$ in length) in intron 2 of the flounder c-type lysozyme gene. These repeated sequences were not observed in other vertebrate c-type lysozyme genes, or in other regions of the flounder lysozyme gene.

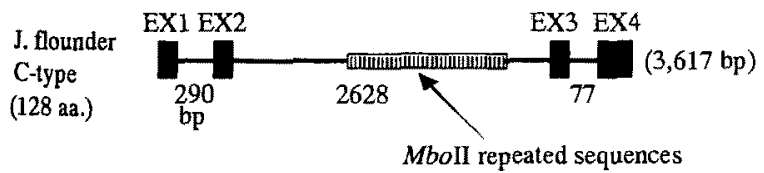

Fig. 1 Structure of the flounder c-type lysozyme gene.

The rainbow trout c-type lysozyme gene is expressed in the liver and kidney, while the Japanese flounder c-type lysozyme gene is expressed in the head kidney, posterior kidney, spleen, brain and ovary Furthermore, in a Japanese flounder injected with Edwardsiella tarda, (a known pathogen of Japanese flounder) and examined after 72 hours, c-type lysozyme mRNA levels in the head kidney, spleen and ovary were greatly increased. ${ }^{7)}$

\section{FLOUNDER G-TYPE LYSOZYME GENE}

The g-type lysozyme gene has also been isolated from Japanese flounder, which is $1,252 \mathrm{bp}$ in length. encoding for 195 amino acid residues. ${ }^{8}$ The Japanese flounder g-type lysozyme gene is about $2.3 \mathrm{~kb}$ longer than the chicken g-type lysozyme gene, this appears to be due to structural differences between the two genes; a 5 exon/4 intron structure in the flounder gene and a 6 exon $/ 5$ intron structure in the chicken gene (Fig. 2) ${ }^{8,9)}$ This difference in length also is partly due to the shortness of the flounder introns relative to the corresponding introns in the chicken gene. In addition, there is a structural differences in exon 1 and intron 1 of the flounder gene relative to the chicken. The $5^{\prime}$ upstream region of the flounder gene must have undergone unknown evolutionary changes, e.g. a recombination, insertion or deletion at the time after higher vertebrates diverged from fish

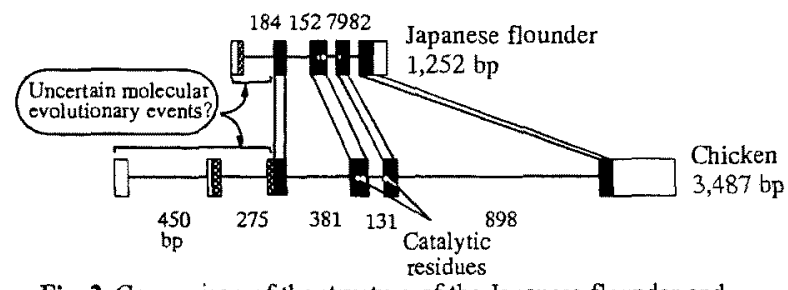

Fig. 2 Comparison of the structure of the Japanese flounder and chicken g-type lysozyme genes.

The Japanese flounder g-type gene is expressed extensively in the head kidney, posterior kidney, spleen, skin, muscle, heart and brain, and also in the liver, intestine, ovary and whole blood to a lesser extent. Furthermore, gene expression in the heart, intestine and whole blood increased following injection with $E$. tarda. ${ }^{8)}$ In contrast, the chicken gtype gene is expressed only in the bone marrow and lung.

\section{THE DIVERSITY OF FISH LYSOZYME}

The c-type, g-type, and phage-type lysozymes were clustered correctly in the phylogenetic analysis (Fig 3 ). It has been proposed that the Japanese flounder gtype lysozyme is closer to the phage-type lysozyme when molecular evolutionary events are considered. 
Potentially the phage-type lysozyme diverged to the flounder g-type lysozyme through the recombination, insertion or deletion of a gene within the $5^{\prime}$ upstream region and that this led to formation of the avian gtype lysozyme. ${ }^{8}$ Gruitter et al. ${ }^{10)}$ hypothesized that the molecular evolution of lysozyme had a common precursor which was like the c-type lysozyme that this gave rise to the g-type lysozyme that led to the present phage-type lysozyme, suggesting that the gtype lysozyme evolved from a phage-type lysozyme, and both of these from a common "c-type like" precursors. However, other authors have proposed that the phage-type and the common precursor of ctype-like lysozyme converged to form the flounder gtype lysozyme. This possibility is based on the similarity between the three dimensional structure of the g-type lysozyme and that of the c-type and phagetype lysozymes. ${ }^{2,11)}$ Further analysis, particularly of g-type lysozyme from a variety of organisms is required to clarify this point.

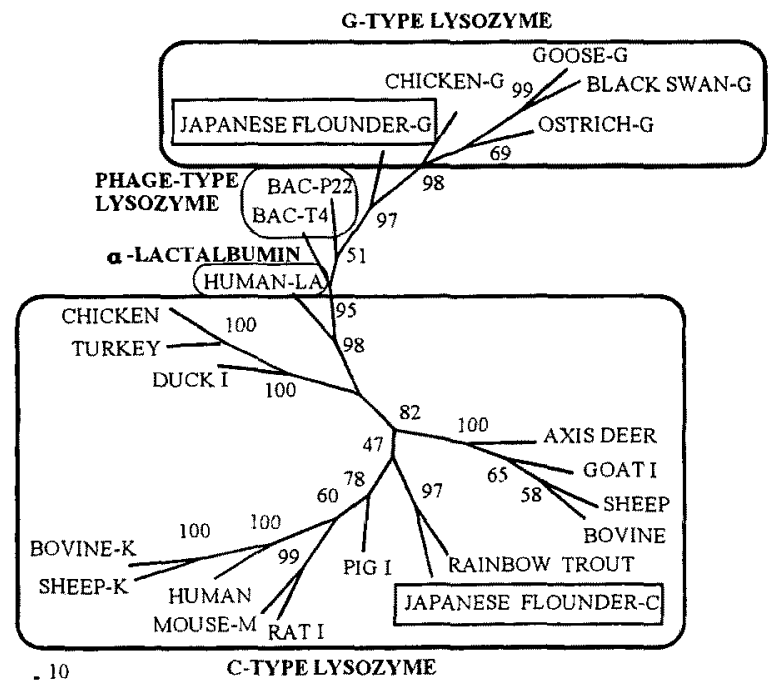

Fig. 3 Phylogenic analysis of Japanese flounder lysozyme.

\section{LYSOZYME ACTIVITY OF JAPANESE FLOUNDER}

It is known that fish lysozyme possess the lytic activity against Micrococcus lysodeikticus, a Grampositive bacteria. ${ }^{212)}$ Rainbow trout lysozyme (type I) has been found to possess substantial antibacterial activity to seven bacterial strains of Gram-negative species. However, hen egg lysozyme was bactericidal only against one species of nonpathogenic bacteria. ${ }^{13)}$ These results suggest that fish lysozyme, in contrast to other higher vertebrate lysozymes, has antibacterial activity not only against Gram-positive bacteria, but also against Gram-negative bacteria. Recombinant ctype and g-type lysozymes of Japanese flounder have been shown to have high bacteriolytic activity against Pasteurella piscicida and Vibrio anguillarum, but low activity against E. tarda and $\beta$-Streptococcus $\mathrm{sp}$ which are pathogenic agents of Japanese flounder. ${ }^{8,4}$; These results indicate that fish lysozyme possess a high potential for bacteriocidal or bacteriolytic activity against nonpathogenic Gram-positive and Gram-negative bacteria, however, they appear to possess a low potential against pathogenic agents

\section{REFERENCES}

1. Yano T. The nonspecific immune system: Humoral defense. In: Iwama $G$. and Nakanishi T (eds). The fish immune system; Organism, Pathogen, and Environment. Academic Press, San Diego. 1996; 105-157.

2. Jollés $P$, Jollés $J$. What is new in lysozyme research? Mol.Cell Biochem. 1984; 63: 165-189.

3. Hikima J, Hirono I, Aoki T. Molecular cloning and novel repeated sequences of a c-type lysozyme gene in Japanese flounder (Paralichthys olivaceus). Mar. Biotechnol. 2000; 2: 241-247.

4. Jollés P (ed). Lysozymes; Model Enzymes in Biochemistry and Biology. Birkhäuser Verlag Basel, Switzerland. 1996.

5. Qasba PK, Kumar S. Molecular divergence of lysozymes and $\alpha$-lactalbumin. Critical Rev. Biochem. Mol. Biol. 1997; 32: 255-306.

6. Dautigny A, Prager EM, Pham-Dinh D, Jollés J, Pakdel F, Grinde B, Jollés P. cDNA and amino acid sequences of rainbow trout (Oncorhynchus mykiss) lysozymes and their implications for the evolution of lysozyme and lactalbumin. J. Mol. Evol. 1991; 32: 187-198.

7. Hikima J, Hirono I, Aoki T. Characterization and expression of c-type lysozyme cDNA from Japanese flounder (Paralichthys olivaceus). Mol. Marine Biol. Biotechnol. 1997; 6: 339-344.

8. Hikima J, Hirono I, Aoki T. Molecular cloning, expression and evolution of the Japanese flounder goose-type lysozyme gene, and the lytic activity of its recombinant protein. Biochim. Biophys. Acta 2001; 1520: 35-44.

9. Nakano T, Graf T. Goose-type lysozyme gene of the chicken: sequence, genomic organization and expression reveals major differences to chicken-type lysozyme gene. Biochim. Biophys. Acta. 1991; 1090: 273-276.

10. Grutter MG, Weaver LH, Matthews BW. Goose lysozyme structure: an evolutionary link between hen and bacteriophage lysozymes? Nature 1983; 303: 828-831.

11. Weaver LH, Grütter MG, Remington SJ, Gray TM, Isaacs NW, Matthews BW. Comparison of goose-type, chicken-type, and phage-type lysozymes illustrates the changes that occur in both amino acid sequence and threedimensional structure during evolution. J. Mol. Evol. 1985; 21 ; $97-111$.

12. Grinde B, Jollés J, Jollés P. Purification and characterization of two lysozymes from rainbow trout (Salmo gairdneri). Eur. J. Biochem. 1988; 173: 269-273.

13. Grinde B. Lysozyme from rainbow trout, Salmo gairdneri Richardson, as an antibacterial agent against fish pathogens. J. Fish Dis. 1989; 12: 95-104.

14. Minagawa S, Hikima J, Hirono I, Aoki T, Mori H. Expression of Japanese flounder cDNA in insect cells. Develop. Comp. immunol. 2001; 25: 439-445 